\title{
Increased Proinflammatory Cytokine Levels in Prolonged Arthralgia in Ross River Virus Infection
}

\section{Dennis Tappe, José Vicente Pérez-Girón, Sergio Gómez-Medina, Stephan Günther, César Muñoz-Fontela, Jonas Schmidt-Chanasit}

Author affiliations: Bernhard Nocht Institute for Tropical Medicine, Hamburg, Germany (D. Tappe, S. Günther, C. Muñoz-Fontela, J. Schmidt-Chanasit); German Centre for Infection Research, Hamburg (D. Tappe, S. Günther, C. Muñoz-Fontela, J. Schmidt-Chanasit); Leibniz Institute for Experimental Virology, Hamburg (J.V. Pérez-Girón, S. Gómez-Medina, C. Muñoz-Fontela)

DOI: http://dx.doi.org/10.3201/eid2304.160466

Ross River virus, a mosquitoborne alphavirus, causes epidemic polyarthritis in Australia and the Pacific region. We analyzed serum cytokine, chemokine, and growth factor levels in travelers returning to Germany from Australia. Serum samples showed elevated concentrations in the acute phase of the illness and, more pronounced, in the long-lasting convalescent phase.

$\mathrm{R}$ oss River virus (RRV) is a mosquitoborne alphavirus endemic to Australia and the Pacific region. The virus is transmitted by various Aedes and Culex spp. mosquito species; macropods are the natural reservoir (1). RRV causes epidemic polyarthritis, with several thousand cases noted annually in Australia (1). Large outbreaks occurred during 1979-1980 on South Pacific islands. Infections in travelers, however, are rare (2). Epidemic polyarthritis is a self-limiting febrile arthralgia syndrome that closely resembles chikungunya and Mayaro fever. Acute-onset symmetric polyarthritis, most often affecting the fingers, wrists, ankles, and knees, is the predominant clinical presentation. Rash, myalgia, fatigue, and fever are present in half of patients (1). Joint effusions are common, and arthralgia can be long-lasting (months to years), and recurring.

The clinical disease, diagnostic procedures, and epidemiology of RRV have been well described, but immunologic parameters in humans and their usefulness in the clinical follow-up of patients warrant further investigation. Therefore, we investigated travelers returning to Germany from Australia with epidemic polyarthritis resulting from RRV infection.

We analyzed 20 serum samples from 16 patients (7 men, 9 women; age range 20-67 years [median 38 years]) ${ }^{1}$ Deceased. who had serologic evidence for acute or recent RRV infection, as confirmed by indirect immunofluorescence assay and virus neutralization assay (2) (Table). All patients had acquired the infection during travel in Australia. We obtained serum samples at different times after symptom onset ( 3 days -16 weeks) and classified them as either acute (taken $\leq 30$ days after symptom onset; $\mathrm{n}=7$ ) or convalescent with symptomatic arthralgia (taken $>30$ days after disease onset; $n=13$ ). After we obtained written consent from the patients, we subjected the serum samples to multiplex cytokine analyses. In parallel with the RRV patients, we tested 20 serum samples from healthy blood donors.

Compared with concentrations in samples from healthy controls, concentrations in samples from patients in the acute phase of RRV infection showed noticeably elevated concentrations of serum interleukin (IL) 4 and 7; granulocyte-macrophage colony stimulating factor (GM$\mathrm{CSF}$ ); regulated on activation, normal $\mathrm{T}$ cell expressed and secreted (RANTES); interferon- $\gamma$-induced protein 10 (IP$10)$; and vascular endothelial growth factor (VEGF). We also saw a notable decrease in eotaxin levels in acute-phase samples. In samples from patients in the arthralgic convalescent phase, we noted increases in IL-1 $\beta$, IL-4, IL-6, IL-8, IL-9, IL-13, IL-15, GM-CSF, interferon- $\gamma$, tumor necrosis factor- $\alpha$ (TNF- $\alpha$ ), RANTES, basic fibroblast growth factor (bFGF), macrophage inflammatory protein $1 \alpha$ (MIP1 $\alpha)$, and VEGF in comparison to healthy controls. Substantial elevations in the convalescent phase when compared with the acute phase were recorded for levels of bFGF and MIP1 $\alpha$, and a marked decrease was seen in IP-10 concentrations.

Cytokine and chemokine levels were generally higher in the convalescent phase than in the acute phase, with individual exceptions (online Technical Appendix, https://wwwnc.cdc.gov/EID/article/23/4/16-0466Techapp1.pdf). No notable changes in either phase were seen for IL-1RA, IL-2, IL-5, IL-10, IL-12p70, IL-17, granulocyte colony-stimulating factor, monocyte chemotactic protein 1 , and platelet-derived growth factor $\beta$ polypeptide levels (data not shown), and macrophage inflammatory protein $1 \beta$ concentrations.

Similar to chikungunya and Mayaro fever, the most prominent clinical symptom of RRV infection is long-lasting arthralgia. Nearly $60 \%$ of patients reported persisting pain after 2-3 years (1). RRV RNA has been detected in synovial fluid up to 5 weeks after symptom onset (3), suggesting ongoing viral replication and inflammation (4). In the RRV-infected travelers examined in this study, the increased proinflammatory cytokine serum concentrations during the prolonged arthralgic convalescence phase strengthen the hypothesis of persisting inflammation of the joints. In patients and in a murine model, macrophage-derived TNF- $\alpha$, interferon- $\gamma$, and IL- 6 were elevated in synovial fluid during RRV disease (5), as we have shown here in serum. 
Table. Returning travelers from Australia infected with Ross River virus included in study of cytokine levels during acute and convalescent disease phases, Germany

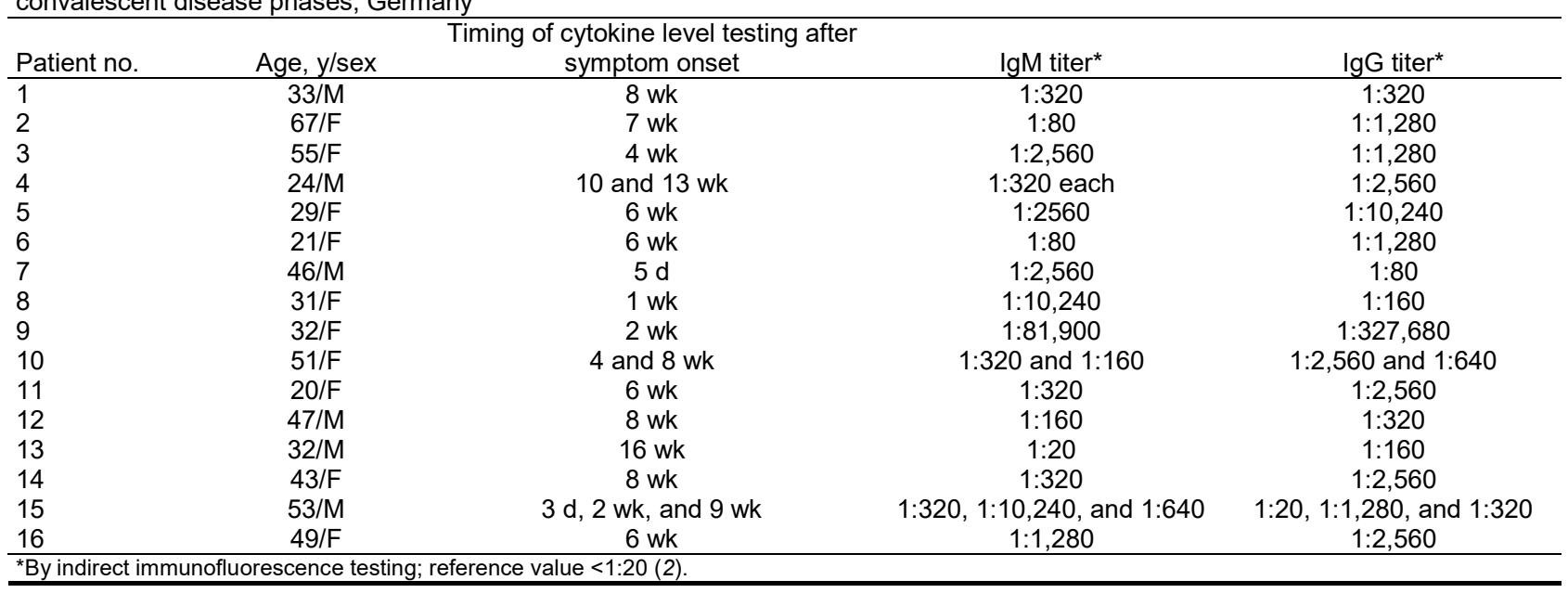

Furthermore, increased levels of IL-1 $\beta$, IL-6, IL-15, MIP1 $\alpha$, and GM-CSF, as seen in our study, have been described in the clinically similar chikungunya (6). Elevated levels of RANTES (and IP-10 initially) indicate T-cell activation, possibly reflecting ongoing viral replication in the joints, also as described in chikungunya (6). A similar effect could recently be demonstrated in Mayaro fever patients with prolonged arthralgia (7).

In conclusion, cytokine level testing in alphavirus infections with prolonged arthralgia may aid monitoring patient symptoms. This information is particularly valuable when clinical signs of arthritis, such as joint swelling and redness, are no longer present, and standard serum inflammatory parameters are within reference ranges. Low-grade inflammation in persistent alphavirus-induced arthritis might place patients at risk for bone loss and fractures (8). Levels of RANKL (receptor activator of nuclear factor $\mathrm{\kappa B}$ ligand), which were not determined in our study, were recently shown to be elevated in RRV infection and associated with increased osteoclast formation (9).

The pattern of cytokine concentration elevations we demonstrated for patients with epidemic polyarthritis is similar to what has recently been described for patients with Mayaro fever (7) and chikungunya $(6,10)$. Our data broaden the knowledge of alphavirus pathogenesis in arthralgia syndromes; however, more immunological investigations, including human T-cell analyses, are needed.

\section{Acknowledgments}

We thank Birgit Hüsing, Corinna Thomé, and Alexander Schlaphof for their excellent technical assistance.

Dr. Tappe is a clinical microbiologist at the National Reference Center for Tropical Diseases, Bernhard Nocht Institute, Hamburg, Germany. His research interests focus on the immune response in viral and rickettsial diseases.

\section{References}

1. Harley D, Sleigh A, Ritchie S. Ross River virus transmission, infection, and disease: a cross-disciplinary review. Clin Microbiol Rev. 2001;14:909-32. http://dx.doi.org/10.1128/ CMR.14.4.909-932.2001

2. Tappe D, Schmidt-Chanasit J, Ries A, Ziegler U, Müller A, Stich A. Ross River virus infection in a traveller returning from northern Australia. Med Microbiol Immunol (Berl). 2009;198:2713. http://dx.doi.org/10.1007/s00430-009-0122-9

3. Soden M, Vasudevan H, Roberts B, Coelen R, Hamlin G, Vasudevan $\mathrm{S}$, et al. Detection of viral ribonucleic acid and histologic analysis of inflamed synovium in Ross River virus infection. Arthritis Rheum. 2000;43:365-9. http://dx.doi.org/10.1002/ 1529-0131(200002)43:2<365::AID-ANR16>3.0.CO;2-E

4. Suhrbier A, La Linn M. Clinical and pathologic aspects of arthritis due to Ross River virus and other alphaviruses. Curr Opin Rheumatol. 2004;16:374-9. http://dx.doi.org/10.1097/01. bor.0000130537.76808.26

5. Lidbury BA, Rulli NE, Suhrbier A, Smith PN, McColl SR, Cunningham AL, et al. Macrophage-derived proinflammatory factors contribute to the development of arthritis and myositis after infection with an arthrogenic alphavirus. J Infect Dis. 2008;197:1585-93. http://dx.doi.org/10.1086/587841

6. Ng LF, Chow A, Sun YJ, Kwek DJ, Lim PL, Dimatatac F, et al. IL-1 $\beta$, IL-6, and RANTES as biomarkers of chikungunya severity. PLoS One. 2009;4:e4261. http://dx.doi.org/10.1371/ journal.pone. 0004261

7. Tappe D, Pérez-Girón JV, Just-Nübling G, Schuster G, Gómez-Medina S, Günther S, et al. Sustained elevated cytokine levels during recovery phase of Mayaro virus infection. Emerg Infect Dis. 2016;22:750-2. http://dx.doi.org/10.3201/eid2204.151502

8. Chen W, Foo SS, Sims NA, Herrero LJ, Walsh NC, Mahalingam S. Arthritogenic alphaviruses: new insights into arthritis and bone pathology. Trends Microbiol. 2015;23:35-43. http://dx.doi.org/ 10.1016/j.tim.2014.09.005

9. Chen W, Foo SS, Rulli NE, Taylor A, Sheng KC, Herrero LJ, et al. Arthritogenic alphaviral infection perturbs osteoblast function and triggers pathologic bone loss. Proc Natl Acad Sci U S A. 2014;111:6040-5. http://dx.doi.org/10.1073/pnas.1318859111

10. Chow A, Her Z, Ong EK, Chen JM, Dimatatac F, Kwek DJ, et al. Persistent arthralgia induced by chikungunya virus infection is associated with interleukin-6 and granulocyte macrophage colony-stimulating factor. J Infect Dis. 2011;203:149-57. http://dx.doi.org/10.1093/infdis/jiq042 
Address for correspondence: Dennis Tappe, Bernhard Nocht Institute for Tropical Medicine, Bernhard-Nocht-Str. 74, 20359 Hamburg, Germany; email: tappe@bnitm.de

\section{Peste des Petits Ruminants Virus in Vulnerable Wild Small Ruminants, Iran, 2014-2016}

\section{Mahmoud Marashi, Siamak Masoudi, Majid Kharazian Moghadam, Hossein Modirrousta, Mahyar Marashi, Masoumeh Parvizifar, Majid Dargi, Mahyar Saljooghian, Farbod Homan, Bernd Hoffmann, Claudia Schulz, Elke Starick, Martin Beer, Sasan Fereidouni}

\begin{abstract}
Author affiliations: Department of Environment, Tehran, Iran (M. Marashi, S. Masoudi, M.K. Moghaddam, M. Marashi, M. Parvizifar, M. Dargi, M. Saljooghian, F. Homan); Razi Research Institute, Karaj, Iran (H. Modirrousta); Friedrich-Loeffler-Institute, Insel Riems, Germany (B. Hoffmann, C. Schulz, E. Starick,

M. Beer); University of Veterinary Medicine Hannover, Hannover, Germany (C. Schulz); University of Veterinary Medicine Vienna, Vienna, Austria (S. Fereidouni)
\end{abstract}

DOI: http://dx.doi.org/10.3201/eid2304.161218

In 2014-2016, >1,000 wild goats and sheep in 4 northern and central provinces of Iran died from peste des petits ruminants virus (PPRV) infection. Partial nucleoprotein sequencing of PPRV from 3 animals showed a close relationship to lineage 4 strains from China. Control measures are needed to preserve vulnerable ruminant populations.

$\mathrm{P}^{2}$ este des petits ruminants virus (PPRV; family Paramyxoviridae, genus Morbillivirus) causes a highly contagious disease with a high death rate in wild and domestic small ruminants. Four PPRV lineages (L1-L4) exist in Africa and Asia (1). The disease was initially recorded in Iran in 1995 (2) and subsequently spread throughout the country (3). PPRV-L4 infections are endemic in Iran and several neighboring countries $(4,5)$.

Wild goats (Capra aegagrus) and sheep (Ovis orientalis), which have become extinct in several West Asia countries, are considered vulnerable species in Iran $(6,7)$. Although PPRV-associated outbreaks among these ruminants have been suspected since 2000 , the virus was not isolated or characterized at that time. In 2001, at least 1,500 wild goats and gazelles (Gazella subgutturosa) with clinical signs similar to those caused by PPRV infection died in Kavir National Park (Figure; online Technical Appendix Table, https://wwwnc.cdc.gov/EID/article/23/4/16-1218-Techapp1.pdf). An estimated $25 \%-40 \%$ of the wild goat population in the park was deemed lost as a result of the disease. In 2011, PPRV was the suspected cause of 550-700 deaths among wild sheep in Sarigol National Park (Figure); laboratory investigations using conventional reverse transcription PCR (RTPCR) confirmed PPRV infection in several dead animals (Iran veterinary organization, pers. comm., 2011 Sep 26).

Beginning in September 2014, park rangers reported and field investigations substantiated mass deaths among wild goats in Bamou National Park (Figure). Clinical signs in affected animals were similar to those reported in wild small ruminants in 2011, and samples we tested from 5 dead goats were positive for PPRV by RT-PCR (online Technical Appendix). In April 2015, a new outbreak started in Haftad Qolleh Arak (Figure) and continued until mid-May, resulting in the death of 428 wild goats and 30 wild sheep. Three more outbreaks occurred in 2015: the first started in August in Kharmaneh-sar Tarom; the second in September in the Alamout Protected Area, $150 \mathrm{~km}$ from the previous outbreak in Kharmaneh-sar Tarom; and the third in November in the Taleghan Protected Area, $100 \mathrm{~km}$ away from the previous outbreak in Alamout Protected Area (Figure; online Technical Appendix Table).

The last reported outbreak started in April 2016 in Khojir, a national park close to a dam that serves as a water source for wild animals (Figure). In 2015, a total of 110 wild goats and sheep were counted in the park, and by May, 1, 2016, $\approx 85$ were found dead (online Technical Appendix Table).

We detected PPRV genome in 6 oral swab samples and 7 blood and lymph node samples from dead ruminants by using conventional RT-PCR and in 3 oral swab samples by using real-time RT-PCR (quantification cycles 31-34) (online Technical Appendix). In addition, we performed partial nucleocapsid gene sequencing of 3 PPRV isolates from 2015; results showed $100 \%$ pairwise nt identity among the isolates (online Technical Appendix). The strains shared highest nt identity (99.4\%) with PPRV-L4 strains that were circulating in domestic or wild small ruminants in northwestern and southeastern China during 2013-2015 (8) (online Technical Appendix Figure); they were more distantly related to PPRV-L4 strains previously reported from outbreaks in Iran and neighboring countries $(9,10)$.

Field investigations and laboratory analyses indicated that PPRV was the cause of mass die-offs of wild goats and sheep during 2014-2016 in several national parks in Iran. A risk assessment of PPRV infection in several developing countries in Africa and the Middle East and on the Indian Peninsula indicated that $63 \%$ of small ruminant populations 